\title{
Citations and Impact of ISI Tourism and Hospitality Journals*
}

\author{
Chia-Lin Chang \\ Department of Applied Economics \\ Department of Finance \\ National Chung Hsing University \\ Taichung, Taiwan \\ Michael McAleer \\ Econometric Institute \\ Erasmus School of Economics \\ Erasmus University Rotterdam \\ and \\ Tinbergen Institute \\ The Netherlands \\ and \\ Institute of Economic Research \\ Kyoto University \\ Japan \\ and \\ Department of Quantitative Economics \\ Complutense University of Madrid \\ Spain
}

\section{EI2011-26}

July 2011

* For financial support, the first author acknowledges the National Science Council, Taiwan; and the second author acknowledges the Australian Research Council, National Science Council, Taiwan, and the Japan Society for the Promotion of Science. 


\begin{abstract}
The paper analyses the leading international journals in Tourism and Hospitality Research using quantifiable Research Assessment Measures (RAMs), highlights the similarities and differences in alternative RAMs, shows that several RAMs capture similar performance characteristics of highly cited journals, and shows that some other RAMs have low correlations with each other, and hence add significant informational value. Several RAMs are discussed for the Thomson Reuters ISI Web of Science database (hereafter ISI). Alternative RAMs may be calculated annually or updated daily to answer the questions as to When, Where and How (frequently) published papers are cited. The RAMs include the most widely used RAM, namely the classic 2-year impact factor including journal self citations (2YIF), 2-year impact factor excluding journal self citations (2YIF*), 5-year impact factor including journal self citations (5YIF), Immediacy (or zero-year impact factor (0YIF)), Eigenfactor, Article Influence, C3PO (Citation Performance Per Paper Online), h-index, PIBETA (Papers Ignored - By Even The Authors), 2-year Self-citation Threshold Approval Ratings (2Y-STAR), Historical Self-citation Threshold Approval Ratings (H-STAR), Impact Factor Inflation (IFI), and Cited Article Influence (CAI). As data are not available for 5YIF, Article Influence and CAI for 11 of the 14 journals considered, 10 RAMs are analysed for 14 highly-cited journals in Tourism and Hospitality in the ISI category of Hospitality, Leisure, Sports \& Tourism. Harmonic mean rankings of the 10 RAMs for the 14 highly-cited journals are also presented. It is shown that emphasizing the 2-year impact factor of a journal, which partly answers the question as to When published papers are cited, to the exclusion of other informative RAMs, which answer Where and How (frequently) published papers are cited, can lead to a distorted evaluation of journal impact.
\end{abstract}

Keywords: Research assessment measures, Impact factor, IFI, C3PO, PI-BETA, STAR, Eigenfactor, Article Influence, h-index.

JEL Classifications: L83, Y10. 


\section{Introduction}

Tourism (including Travel) and Hospitality is one of the world's most important industries in terms of overall GDP and the number of individuals who are employed in the sector, both directly and indirectly. This area is also of significant interest to researchers and policy analysts. Therefore, it is not surprising that the number of international journals in Tourism and Hospitality has increased considerably in recent years. However, the growing number of international journals in Tourism and Hospitality has not yet been analysed in terms of citations and impact in the academic literature.

Publishing high quality research is fundamental to scientific progress. Consequently, it is essential to evaluate the research output and impact of individual researchers. The perceived research performance of individual researchers can be crucial for hiring, promotion and tenure decisions in many countries worldwide. In the absence of appropriate information regarding the perceived quality of an individual's research output, the perceived quality of academic journals, however measured, has frequently been used as a guide, albeit inexact, for determining the quality of academic research publications.

It is well known that the perceived quality of a journal can be an inappropriate and misleading proxy for the perceived quality of a paper that has been published in the journal. The quality and impact of an academic journal are typically based on outstanding papers that have already been published in the journal. However, publication in a leading journal should not be taken to be an accurate reflection of the quality of a published paper, especially when the paper has not yet received many, or any, citations. In this regard, Seglen (1997) finds that, although the citations rates of published papers determine the impact factor of journals, the reverse does not hold.

Leading journals publish important papers, though they may not publish many important papers. Such journals typically increase the visibility of the research findings of published papers, which may subsequently lead to higher citations. Otherwise, there would seem to be little point in publishing in leading journals, especially as the primary purpose in writing papers is to be cited, rather than just being published. 
The acceptance of a paper for publication in a journal is typically based on the purported expertise of a few members of the Editorial Board and referees. These professionals determine the rejection rate of a journal before publication. However, members of editorial boards and referees can, and do, make mistakes. After a paper has been published in a journal, the implicit rejection rate depends on the worldwide academic professional community. Consequently, the proportion of published papers that is ignored by the profession, and by even the authors, is an important (non-) impact performance measure after publication. The community of academic scholars worldwide is less likely to make serious errors regarding the quality of academic research papers after they have been published, especially after several years have passed, than a small group of Editorial Board members and referees who are required to make (frequently incorrect) judgments regarding the quality of a paper before it is published in a journal.

Citations capture both the impact of a journal and the impact or performance of individual researchers. Citations should be, and are, more important than publications for individual researchers, especially in the sciences. As the only quantitative way in which to evaluate journal impact is through citations, it is not surprising that all RAMs are based on citations. The Thomson Reuters ISI Web of Science (2011) database (hereafter ISI) is a leading high quality database for generating RAMs to evaluate the research performance of individual researchers and the quality of academic journals (see Seglen (1997) and Chang et al. (2011a, b, c), among others, for caveats regarding ISI data).

This paper examines the importance of RAMs as viable rankings criteria in Tourism and Hospitality, attempts to answer the questions as to When, Where and How (frequently) published papers are cited in leading Tourism and Hospitality journals, and evaluates the usefulness of 10 existing RAMs for 14 leading journals in Tourism and Hospitality in the ISI category of Hospitality, Leisure, Sports \& Tourism.

The plan of the remainder of the paper is as follows. Section 2 presents some key RAM using ISI data that may be calculated annually or updated daily, including the most widely used RAM, namely the classic 2-year impact factor including journal self citations (2YIF), 2-year impact factor excluding journal self citations (2YIF*), 5-year impact factor including journal self citations (5YIF), Immediacy (or zero-year impact factor (0YIF)), Eigenfactor, Article Influence, C3PO (Citation Performance Per Paper Online), h-index, PI-BETA (Papers 
Ignored - By Even The Authors), 2-year Self-citation Threshold Approval Ratings (2YSTAR), Historical Self-citation Threshold Approval Ratings (H-STAR), Impact Factor Inflation (IFI), and Cited Article Influence (CAI). Section 3 discusses and analyses 10 RAMs for 14 leading journals in Tourism and Hospitality in the ISI category of Hospitality, Leisure, Sports \& Tourism. Section 4 summarizes the outcomes.

\section{Research Assessment Measures (RAM)}

The RAMs are intended as descriptive statistics to capture journal impact and performance, and are not based on a mathematical model. Hence, in what follows, no optimization or estimation is required in calculating the alternative RAMs.

As the alternative RAMs that are provided in ISI and in several recent publications may not be widely known, this section provides a brief description and definition of 13 RAMs that may be calculated annually or updated daily to answer the questions as to When, and Where and How (frequently), published papers are cited. The answers to When published papers are cited are based on $\{2$ YIF, 2YIF*, 5YIF, Immediacy , and the answers to Where and How (frequently) published papers are cited are based on \{Eigenfactor, Article Influence, IFI, HSTAR, 2Y-STAR, C3PO, h-index, PI-BETA, CAI\}.

\subsection{Annual RAM}

With two exceptions, namely Eigenfactor and Article Influence, existing RAMs are reported separately for the sciences and social sciences. RAMs may be computed annually or updated daily. The annual RAMs given below are calculated for a Journal Citations Reports (JCR) calendar year, which is the year before the annual RAM are released. For example, the RAMs were released in late-June 2011 for the JCR calendar year 2010.

\section{(1) 2-year impact factor including journal self citations (2YIF):}

The classic 2-year impact factor including journal self citations (2YIF) of a journal is typically referred to as "the impact factor", is calculated annually, and is defined as "Total 
citations in a year to papers published in a journal in the previous 2 years / Total papers published in a journal in the previous 2 years”. The choice of 2 years by ISI is arbitrary.

\section{(2) 2-year impact factor excluding journal self citations (2YIF*):}

ISI also reports a 2-year impact factor without journal self citations (that is, citations to a journal in which a citing paper is published), which is calculated annually. As this impact factor is not widely known or used, Chang et al. (2011c) refer to this RAM as 2YIF*.

\section{(3) 5-year impact factor including journal self citations (5YIF):}

The 5-year impact factor including journal self citations (5YIF) of a journal is calculated annually, and is defined as "Total citations in a year to papers published in a journal in the previous 5 years / Total papers published in a journal in the previous 5 years.” The choice of 5 years by ISI is arbitrary.

\section{(4) Immediacy, or zero-year impact factor including journal self citations (OYIF):}

Immediacy is a zero-year impact factor including journal self citations (0YIF) of a journal, is calculated annually, and is defined as "Total citations to papers published in a journal in the same year / Total papers published in a journal in the same year." The choice of the same year by ISI is arbitrary.

\section{(5) Eigenfactor:}

The Eigenfactor score (see Bergstrom (2007), Bergstrom and West (2008), Bergstrom, West and Wiseman (2008)) is a modified 5YIF, and is calculated annually. The Eigenfactor algorithm (see www.eigenfactor.org/methods.htm) effectively ranks journals according to citations and the length of time that researchers are logged on to a journal's website. To state the obvious, the Eigenfactor does not check how much time researchers spend reading hard copies of journals.

\section{(6) Article Influence:}

Article Influence (see Bergstrom (2007), Bergstrom and West (2008), Bergstrom, West and Wiseman (2008)) measures the relative importance of a journal on a per-article basis, is a standardized Eigenfactor score, and is calculated annually. Article Influence is defined as "Eigenfactor score divided by the fraction of all articles published by a journal." 


\section{(7) IFI:}

The ratio of 2YIF to 2YIF* is intended to capture how journal self citations inflate the impact factor of a journal. Chang et al. (2011a) define Impact Factor Inflation (IFI) as "IFI = 2YIF / 2YIF*”. The minimum value for IFI is 1 , with any value above the minimum capturing the effect of journal self citations on the 2-year impact factor.

\section{(8) H-STAR:}

ISI has implicitly recognized the inflation in journal self citations by calculating an impact factor that excludes self citations, and provides data on journal self citations, both historically and for the preceding two years, in calculating 2YIF. Chang et al. (2011b) define the Selfcitation Threshold Approval Rating (STAR) as the percentage difference between citations in other journals and journal self citations. Thus, if $\mathrm{HS}=$ historical journal self citations, $\mathrm{H}$ STAR is defined as “H-STAR = [(100-HS) $-\mathrm{HS}]=(100-2 \mathrm{HS})$ ”. If HS = 0 (minimum), 25, 50 or 100 (maximum) percent, for example, H-STAR = 100, 50, 0 and -100, respectively.

\section{(9) 2Y-STAR:}

If $2 \mathrm{YS}=$ journal self citations over the preceding 2-year period, $2 \mathrm{Y}$-STAR is defined as " $2 \mathrm{Y}$ STAR $=[(100-2 Y S)-2 Y S]=(100-2(2 Y S)) "$. Thus, if 2YS = 0 (minimum), 25, 50 or 100 (maximum) percent, for example, 2Y-STAR = 100, 50, 0 and -100 , respectively.

\subsection{Daily Updated RAM}

Some RAMs are updated daily, and are reported for a given day in a calendar year rather than for a JCR year.

\section{(10) C3PO:}

ISI reports the mean number of citations for a journal, namely total citations up to a given day divided by the number of papers published in a journal up to the same day, as the "average" number of citations. In order to distinguish the mean from the median and mode, the C3PO of an ISI journal on any given day is defined by Chang et al. (2011a) as "C3PO (Citation Performance Per Paper Online) = Total citations to a journal / Total papers published in a journal.” [Note: C3PO should not be confused with C-3PO, the Star Wars android.]

\section{(11) h-index:}


The h-index (Hirsch, 2005)) was originally proposed to assess the scientific research productivity and citations impact of individual researchers. However, the h-index can also be calculated for journals, and should be interpreted as assessing the impact or influence of highly cited journal publications. The h-index of a journal on any given day is based on cited and citing papers, including journal self citations, and is defined as "h-index = number of published papers, where each has at least h citations.”

\section{(12) PI-BETA:}

This RAM measures the proportion of papers in a journal that has never been cited, As such, PI-BETA is, in effect, a rejection rate of a journal after publication. Chang et al. (2011c) argue that lack of citations of a published paper, especially if it is not a recent publication, reflects on the quality of a journal by exposing: (i) what might be considered as incorrect decisions by the members of the editorial board of a journal; and (ii) the lost opportunities of papers that might have been cited had they not been rejected by the journal. Chang et al. (2011c) propose that a paper with zero citations in ISI journals can be measured by PI-BETA (= Papers Ignored (PI) - By Even The Authors (BETA)), which is calculated for an ISI journal on any given day as "Number of papers with zero citations in a journal / Total papers published in a journal.”

\section{(13) CAI:}

Article Influence is intended to measure the average influence of an article across the sciences and social sciences. As an article with zero citations cannot have influence, a more suitable measure of the influence of cited articles is Cited Article Influence (CAI). Chang et al. (2011b) define CAI as “CAI = (1 - PI-BETA)(Article Influence)”. If PI-BETA $=0$, then $\mathrm{CAI}$ is equivalent to Article Influence; if PI-BETA $=1$, then CAI $=0$. As Article Influence is calculated annually and PI-BETA is updated daily, CAI may be updated daily.

\section{Analysis of RAMs for ISI Journals in Tourism and Hospitality}

The ISI category of Hospitality, Leisure, Sports \& Tourism has 31 journals, 14 of which cover Tourism (including Travel) and Hospitality, including 3 journals that have both Tourism and Hospitality in their titles, with the remaining 17 journals covering Sports and 
Leisure. Many of the journals are recent inclusions in ISI, with 15 of the 31 journals having been included for less than 5 years. As 5YIF, Article Influence and CAI data are not available for 11 of the 14 journals in Tourism and Hospitality (the three exceptions being Tourism Management, Annals of Tourism Research, and Cornell Hospitality Quarterly), the 10 available RAMs are analysed for 14 leading Tourism and Hospitality journals in the ISI category of Hospitality, Leisure, Sports \& Tourism.

In Table 1 we evaluate the 14 most highly-cited journals, which are ranked according to 2YIF, in Tourism and Hospitality. Only articles from the ISI Web of Science are included in the citations data. The citations data for all journals were downloaded from ISI on 2 July 2011 for 1988-2010, so that citations were counted from 1988 for all papers that have been published in an ISI journal since 1988.

Five of the 14 journals are listed in more than the ISI category of Hospitality, Leisure, Sports \& Tourism. The additional ISI categories for these 5 journals are given below:

(1) Tourism Management: Environmental Studies; Management;

(2) Annals of Tourism Research: Sociology;

(3) Cornell Hospitality Quarterly: Management; Sociology;

(4) Scandinavian Journal of Hospitality \& Tourism: Sociology;

(5) Journal of Hospitality Leisure Sport \& Tourism Education: Education and Educational Research.

As can be seen from Table 1, the means and ranges of 2YIF are, respectively, 1.014 and $(0.250,2.620)$, of $2 \mathrm{YIF}^{*}$ are 0.723 and $(0.062,2.135)$, and of Immediacy are 0.172 and (0, 0.417). These impact factors seem to be rather low, especially in comparison with the related areas of economics, finance, management, and marketing (see Chang et al. (2011a)), and especially the sciences (see Chang et al. (2011b)). Journal self citations in Tourism and Hospitality seem relatively high, with a mean IFI of 1.821 and a range of $(1.000,4.304)$, with the second highest IFI being 4.032. This means that 2 of the 14 journals in Tourism and Hospitality have 2YIF that is inflated by a factor of greater than 4 through self citations.

The h-index has a mean of 10.36 and a range of $(3,46)$, with only 2 journals having hindexes in double figures, while C3PO has a low mean of 1.73 and a range of $(0.49,7.44)$. In general, papers published in the 14 leading Tourism and Hospitality journals do not seem to 
be well cited. Eigenfactor has a mean of 0.00123 and a range of $(0.00003,0.00599)$, with Tourism Management having by far the highest score, and hence the greatest influence, in Tourism and Hospitality.

H-STAR and 2Y-STAR for the 14 journals are remarkably high, with a mean of 48.57 and a range of $(-22,80)$ for H-STAR, and a mean of 33.43 and a range of $(-52,100)$ for 2Y-STAR. The H-STAR and 2Y-STAR means of 48.57 and 33.43 reflect journal self citations of 26\% and 33\%, respectively, historically and for the preceding two years. For most journals, self citations have increased over the preceding two years as compared with historical levels. One of the few exceptions is the Journal of Hospitality \& Tourism Research, which historically has had $17 \%$ journals self citations, but had zero self citations in the preceding two years. Although there is no suggestion that authors and/or journal editors are being encouraged to increase citations through journal self citations, the prevailing situation might be addressed by encouraging authors to cite from a wider range of Tourism and Hospitality journals than would presently seem to be the case.

The PI-BETA outcomes are revealing. The mean is 0.591 so that, on average, 3 of every 5 papers that are published in the leading 14 journals in Tourism and Hospitality are not cited. The range of $(0.402,0.763)$ suggests that the journal with the highest percentage of cited papers, Tourism Management, has 2 uncited papers for every 5 published papers, while the journal with the lowest percentage of cited papers, Cornell Hospitality Quarterly, has 3 uncited papers for every 4 published papers. It should be emphasized that these are the 14 leading journals in Tourism and Hospitality.

Even taking account of the fact that 11 of the 14 journals in Tourism and Hospitality have been included in the ISI database for less than 5 years, the PI-BETA scores are very high in relation to economics, finance, management and marketing (see Chang et al. (2011a)), and especially in comparison with the sciences (see Chang et al. (2011b)). As it is widely held, especially in the sciences, that the primary purpose in writing papers is to be cited, and not just published, much work needs to be done to increase the citations in the leading Tourism and Hospitality journals.

The simple correlations of the 10 RAMs for the 14 leading journals in Tourism and Hospitality are given in Table 2. The 5 RAM pairs for which the correlations exceed 0.9 (in 
absolute value) are (h-index, C3PO), (2YIF, 2YIF*), (IFI, 2Y-STAR), (2YIF*, Eigenfactor), and (2YIF, Eigenfactor). There are 9 RAM pairs for which the simple correlations are in the range $(0.8,0.9)$, in absolute value. The correlation of 0.997 between the h-index and C3PO is extremely high, which suggests that the mean number of citations and the h-index of a journal are very similar, at least for journals in Tourism and Hospitality.

Fersht (2009) showed that there was a very strong positive correlation between Eigenfactor and the total number of journal citations, with a correlation coefficient of 0.968 for the top 200 cited ISI journals in 2007. Such a high correlation is not entirely surprising as it captures the size effect of journals, with the total number of publications and total citations typically being positively and highly correlated.

It remains to be seen whether an emphasis on the classic 2-year impact factor of a journal, to the virtual exclusion of other informative RAMs, can lead to a distorted evaluation of journal impact and influence. In order to summarize the 10 RAMs, 6 of which, namely 2YIF, 2YIF*, Immediacy, IFI, C3PO and PI-BETA, are based on ratios, the rankings of the 14 journals in Tourism and Hospitality given in Table 3 are based on the harmonic mean, which is given in the last column as Rank.

Compared with the rankings in Table 1 that were based on 2YIF, only the first three journals, namely Tourism Management, Annals of Tourism Research, and Journal of Travel Research, the number 9 ranked journal, Current Issues in Tourism, and the number 14 ranked journal, Journal of Hospitality Leisure Sport \& Tourism Education, remain unchanged in Table 3. Three journals to have moved up considerably are Journal of Hospitality \& Tourism Research (from 8 to 4), Current Issues in Tourism (from 12 to 7), and Scandinavian Journal of Hospitality and Tourism (from 13 to 10). Four journals dropped by only one rank position, but Journal of Travel \& Tourism Marketing moved appreciably in the opposite direction (from 6 to 12).

It is clear that the use of the harmonic mean penalizes widely-varying rankings across the 10 RAMs, as in the case of Journal of Travel \& Tourism Marketing (which was ranked number 6 according to one RAM, and number 14 according to two RAMs). Equivalently, the harmonic mean rewards journals with strong individual performances according to alternative RAMs, so that even one very strong performance can lead to a high, or greatly improved, 
ranking. This is the case for Journal of Hospitality \& Tourism Research (which was ranked number 1 according to 2 RAMs), Current Issues in Tourism (which was ranked number 2 according to one RAM, and number 4 according to two RAMs), and Scandinavian Journal of Hospitality and Tourism (which was ranked number 6 according to two RAMs, and number 7 according to three RAMs).

The simple ranking correlations of the 10 RAMs for the 14 leading journals in Tourism and Hospitality, based on the rankings in Table 3, are given in Table 2. The ranking correlation of 1 for the RAM pair (IFI, 2Y-STAR) suggests that the ranking of the 14 journals is identical based on these 2 RAMs. However, the ranking correlation of 0.659 for the RAM pairs (IFI, H-STAR) and (2Y-STAR, H-STAR) suggests that the inflation in impact factor through journal self citations has been quite different historically from what has been observed over the preceding two years. The ranking correlation of 0.925 for the RAM pair ((2YIF, Eigenfactor) suggests that the classic two-year impact factor is highly correlated with journal influence, as measured by Eigenfactor. The ranking correlation of 0.810 for the RAM pair (hindex, CЗPO) is much lower than the correlation of 0.997 for the same RAM pair in terms of the raw data for the h-index and the mean number of citations across the 14 leading journals in Tourism and Hospitality.

\section{Conclusion}

This paper analysed the leading journals in Tourism and Hospitality using quantifiable Research Assessment Measures (RAMs). Alternative RAMs were discussed for the Thomson Reuters ISI Web of Science (2011) database (hereafter ISI). Ten RAMs were analysed for 14 highly-cited journals in Tourism and Hospitality in the ISI category of Hospitality, Leisure, Sports \& Tourism. The 10 RAMs that may be calculated annually or updated daily answer the questions as to When, and Where and How (frequently), published papers are cited. The answers to When published papers are cited are based on $\left\{2 \mathrm{YIF}, 2 \mathrm{YIF}^{*}\right.$, Immediacy $\}$, and the answers to Where and How (frequently) published papers are cited are based on \{Eigenfactor, IFI, H-STAR, 2Y-STAR, C3PO, h-index, PI-BETA\}. 
The paper highlighted the similarities and differences in alternative RAMs, and showed that several RAMs were highly correlated with existing RAMs, so that they had little informative incremental value in capturing the impact and performance of the highly-cited journals, whereas other RAMs were not highly correlated, thereby providing additional information about journal impact.

Harmonic mean rankings of the 10 RAM were also presented for these 14 leading journals in Tourism and Hospitality. It was shown that emphasizing the 2-year impact factor of a journal, which partly answers the question as to When published papers are cited, to the exclusion of other informative RAMs, which answer Where and How (frequently) published papers are cited, can lead to a distorted evaluation of journal impact. Therefore, the harmonic mean rankings provide a more robust measure of citations and impact than relying solely on the 2year impact factor. 


\section{References}

Bergstrom C. (2007), Eigenfactor: Measuring the value and prestige of scholarly journals, C\&RL News, 68, 314-316.

Bergstrom, C.T. and. J.D. West (2008), Assessing citations with the Eigenfactor ${ }^{\mathrm{TM}}$ metrics, Neurology, 71, 1850-1851.

Bergstrom, C.T., J.D. West and M.A. Wiseman (2008), The Eigenfactor ${ }^{\mathrm{TM}}$ metrics, Journal of Neuroscience, 28(45), 11433-11434 (November 5, 2008).

Chang, C.-L., M. McAleer and L. Oxley (2011a), What makes a great journal great in economics? The singer not the song, Journal of Economic Surveys, 25(2), 326-361.

Chang, C.-L., M. McAleer and L. Oxley (2011b), What makes a great journal great in the sciences? Which came first, the chicken or the egg?, Scientometrics, 87(1), 17-40.

Chang, C.-L., M. McAleer and L. Oxley (2011c), Great expectatrics: Great papers, great journals, great econometrics, Econometric Reviews, 30(6), 583-619.

Fersht, A. (2009), The most influential journals: Impact factor and Eigenfactor, Proceedings of the National Academy of Sciences of the United States of America, 106(17), 6883-6884 (April 28, 2009).

Hirsch, J.E. (2005), An index to quantify an individual's scientific research output, Proceedings of the National Academy of Sciences of the United States of America, 102(46), 16569-15572 (November 15, 2005).

ISI Web of Science (2011), Journal Citation Reports, Essential Science Indicators, Thomson Reuters ISI.

Seglen, P.O. (1997), Why the impact factor of journals should not be used for evaluating research, BMJ: British Medical Journal, 314(7079), 498-502. 
Table 1

Research Assessment Measures (RAM) for14 Tourism and Hospitality Journals

\begin{tabular}{|c|c|c|c|c|c|c|c|c|c|c|}
\hline Journal & 2YIF & 2YIF* & IFI & Immediacy & h-index & C3PO & PI-BETA & Eigenfactor & H-STAR & 2Y-STAR \\
\hline Tourism Management & 2.620 & 2.135 & 1.227 & 0.305 & 39 & 6.18 & 0.402 & 0.00599 & 76 & 64 \\
\hline Annals of Tourism Research & 1.949 & 1.397 & 1.395 & 0.212 & 46 & 7.44 & 0.405 & 0.00281 & 78 & 44 \\
\hline Journal of Travel Research & 1.549 & 1.305 & 1.187 & 0.316 & 7 & 1.61 & 0.503 & 0.00203 & 80 & 70 \\
\hline Journal of Sustainable Tourism & 1.539 & 1.145 & 1.344 & 0.417 & 7 & 1.34 & 0.518 & 0.00146 & 52 & 50 \\
\hline International Journal of Hospitality Management & 1.382 & 0.944 & 1.464 & 0.115 & 9 & 1.50 & 0.533 & 0.00108 & 60 & 38 \\
\hline Journal of Travel \& Tourism Marketing & 0.835 & 0.194 & 4.304 & 0.054 & 4 & 0.63 & 0.675 & 0.00052 & 4 & -52 \\
\hline International Journal of Tourism Research & 0.802 & 0.616 & 1.302 & 0.049 & 4 & 0.70 & 0.600 & 0.00079 & 72 & 54 \\
\hline Journal of Hospitality \& Tourism Research & 0.653 & 0.653 & 1.000 & 0.038 & 5 & 0.83 & 0.624 & 0.00048 & 66 & 100 \\
\hline Tourism Geographies & 0.633 & 0.429 & 1.476 & 0.320 & 4 & 0.78 & 0.649 & 0.00042 & 72 & 36 \\
\hline Tourism Economics & 0.614 & 0.406 & 1.512 & 0.016 & 5 & 0.65 & 0.698 & 0.00081 & 32 & 32 \\
\hline Cornell Hospitality Quarterly & 0.549 & 0.211 & 2.602 & 0.056 & 4 & 0.49 & 0.763 & 0.00008 & 4 & -22 \\
\hline Current Issues in Tourism & 0.542 & 0.424 & 1.278 & 0.344 & 4 & 0.52 & 0.686 & 0.00044 & 48 & 58 \\
\hline Scandinavian Journal of Hospitality and Tourism & 0.282 & 0.205 & 1.376 & 0.160 & 4 & 0.98 & 0.549 & 0.00025 & 58 & 46 \\
\hline Journal of Hospitality Leisure Sport \& Tourism Education & 0.250 & 0.062 & 4.032 & 0.000 & 3 & 0.57 & 0.675 & 0.00003 & -22 & -50 \\
\hline Mean & 1.014 & 0.723 & 1.821 & 0.172 & 10.36 & 1.73 & 0.591 & 0.00123 & 48.57 & 33.43 \\
\hline
\end{tabular}

Note: Ranked according to 2YIF. Citations data were downloaded from ISI on 2 July 2011 for 1988-2010. 
Table 2

Correlations of 10 RAMs for 14 Tourism and Hospitality Journals

\begin{tabular}{|c|c|c|c|c|c|c|c|c|c|c|}
\hline RAM & 2YIF & 2 YIF* $^{*}$ & IFI & Immediacy & h-index & CЗPO & PI-BETA & Eigenfactor & H-STAR & 2Y-STAR \\
\hline 2 YIF & 1.000 & & & & & & & & & \\
\hline $2 Y I^{*}$ & 0.969 & 1.000 & & & & & & & & \\
\hline IFI & -0.349 & -0.521 & 1.000 & & & & & & & \\
\hline Immediacy & 0.502 & 0.549 & -0.487 & 1.000 & & & & & & \\
\hline h-index & 0.811 & 0.774 & -0.248 & 0.277 & 1.000 & & & & & \\
\hline СЗРО & 0.823 & 0.792 & -0.267 & 0.309 & 0.997 & 1.000 & & & & \\
\hline PI-BETA & -0.846 & -0.870 & 0.471 & -0.491 & -0.771 & -0.811 & 1.000 & & & \\
\hline Eigenfactor & 0.926 & 0.931 & -0.334 & 0.433 & 0.834 & 0.838 & -0.792 & 1.000 & & \\
\hline H-STAR & 0.542 & 0.661 & -0.878 & 0.544 & 0.421 & 0.455 & -0.690 & 0.492 & 1.000 & \\
\hline 2Y-STAR & 0.361 & 0.548 & -0.948 & 0.453 & 0.232 & 0.255 & -0.504 & 0.359 & 0.883 & 1.000 \\
\hline
\end{tabular}

Note: Citations data were downloaded from ISI on 2 July 2011 for 1988-2010. 
Table 3

Harmonic Mean Rankings of 10 RAMs for 14 Tourism and Hospitality Journals

\begin{tabular}{|c|c|c|c|c|c|c|c|c|c|c|c|}
\hline Journal & 2YIF & 2YIF* & IFI & Immediacy & h-index & CЗPO & PI-BETA & Eigenfactor & H-STAR & 2Y-STAR & Rank \\
\hline Tourism Management & 1 & 1 & 3 & 5 & 2 & 2 & 1 & 1 & 3 & 3 & 1 \\
\hline Annals of Tourism Research & 2 & 2 & 8 & 6 & 1 & 1 & 2 & 2 & 2 & 8 & 2 \\
\hline Journal of Travel Research & 3 & 3 & 2 & 4 & 4 & 3 & 3 & 3 & 1 & 2 & 3 \\
\hline Journal of Hospitality \& Tourism Research & 8 & 7 & 1 & 12 & 6 & 7 & 8 & 9 & 6 & 1 & 4 \\
\hline Journal of Sustainable Tourism & 4 & 4 & 6 & 1 & 4 & 5 & 4 & 4 & 9 & 6 & 5 \\
\hline International Journal of Hospitality Management & 5 & 5 & 9 & 8 & 3 & 4 & 5 & 5 & 7 & 9 & 6 \\
\hline Current Issues in Tourism & 12 & 9 & 4 & 2 & 8 & 13 & 12 & 10 & 10 & 4 & 7 \\
\hline International Journal of Tourism Research & 7 & 6 & 5 & 11 & 8 & 9 & 7 & 7 & 4 & 5 & 8 \\
\hline Tourism Geographies & 9 & 8 & 10 & 3 & 8 & 8 & 9 & 11 & 4 & 10 & 9 \\
\hline Scandinavian Journal of Hospitality and Tourism & 13 & 12 & 7 & 7 & 8 & 6 & 6 & 12 & 8 & 7 & 10 \\
\hline Tourism Economics & 10 & 10 & 11 & 13 & 6 & 10 & 13 & 6 & 11 & 11 & 11 \\
\hline Journal of Travel \& Tourism Marketing & 6 & 13 & 14 & 10 & 8 & 11 & 10 & 8 & 12 & 14 & 12 \\
\hline Cornell Hospitality Quarterly & 11 & 11 & 12 & 9 & 8 & 14 & 14 & 13 & 12 & 12 & 13 \\
\hline Journal of Hospitality Leisure Sport \& Tourism Education & 14 & 14 & 13 & 14 & 14 & 12 & 10 & 14 & 14 & 13 & 14 \\
\hline
\end{tabular}

Note: Ranked according to harmonic mean (Rank). Citations data were downloaded from ISI on 2 July 2011 for $1988-2010$. 
Table 4

Ranking Correlations of 10 RAMs for 14 Tourism and Hospitality Journals

\begin{tabular}{|c|c|c|c|c|c|c|c|c|c|c|}
\hline RAM & 2YIF & 2YIF* & IFI & Immediacy & h-index & C3PO & PI-BETA & Eigenfactor & H-STAR & 2Y-STAR \\
\hline 2YIF & 1.000 & & & & & & & & & \\
\hline 2 YIF $^{*}$ & 0.864 & 1.000 & & & & & & & & \\
\hline IFI & 0.380 & 0.657 & 1.000 & & & & & & & \\
\hline Immediacy & 0.376 & 0.512 & 0.389 & 1.000 & & & & & & \\
\hline h-index & 0.854 & 0.865 & 0.457 & 0.452 & 1.000 & & & & & \\
\hline СЗРО & 0.785 & 0.820 & 0.495 & 0.380 & 0.810 & 1.000 & & & & \\
\hline PI-BETA & 0.780 & 0.798 & 0.526 & 0.419 & 0.688 & 0.945 & 1.000 & & & \\
\hline Eigenfactor & 0.925 & 0.868 & 0.442 & 0.345 & 0.887 & 0.789 & 0.744 & 1.000 & & \\
\hline H-STAR & 0.686 & 0.828 & 0.659 & 0.419 & 0.665 & 0.788 & 0.751 & 0.641 & 1.000 & \\
\hline 2Y-STAR & 0.380 & 0.657 & 1.000 & 0.389 & 0.457 & 0.495 & 0.526 & 0.442 & 0.659 & 1.000 \\
\hline
\end{tabular}

Note: Citations data were downloaded from ISI on 2 July 2011 for 1988-2010. 